\title{
Cerebrospinal fluid and plasma cytokines after subarachnoid haemorrhage: CSF interleukin- 6 may be an early marker of infection
}

Stephen J Hopkins ${ }^{1 *}$, Catherine J McMahon ${ }^{3}$, Navneet Singh ${ }^{1}$, James Galea', Margaret Hoadley ${ }^{1}$, Sylvia Scarth , Hiren Patel ${ }^{1}$, Andy Vail ${ }^{1}$, Sharon Hulme ${ }^{1}$, Nancy J Rothwell ${ }^{2}$, Andrew T King ${ }^{1}$ and Pippa J Tyrrell ${ }^{1}$

\begin{abstract}
Background: Cytokines and cytokine receptor concentrations increase in plasma and cerebrospinal fluid (CSF) of patients following subarachnoid haemorrhage (SAH). The relationship between plasma and CSF cytokines, and factors affecting this, are not clear.

Methods: To help define the relationship, paired plasma and cerebrospinal fluid (CSF) samples were collected from patients subject to ventriculostomy. Concentrations of key inflammatory cytokines, interleukin (IL)-1ß, IL-1 receptor antagonist (IL-1Ra), IL-1 receptor 2, IL-6, IL-8, IL-10, tumour necrosis factor (TNF)-a, and TNF receptors (TNF-R) 1 and 2 were determined by immunoassay of CSF and plasma from 21 patients, where samples were available at three or more time points.
\end{abstract}

Results: Plasma concentrations of IL-1ß, IL-1Ra, IL-10, TNF- $a$ and TNF-R1 were similar to those in CSF. Plasma TNF-R2 and IL-1R2 concentrations were higher than in CSF. Concentrations of IL-8 and IL-6 in CSF were approximately 10 to 1,000-fold higher than in plasma. There was a weak correlation between CSF and plasma IL-8 concentrations $(r=0.26)$, but no correlation for IL-6. Differences between the central and peripheral pattern of IL-6 were associated with episodes of ventriculostomy-related infection (VRI). A VRI was associated with CSF IL-6 $>10,000 \mathrm{pg} / \mathrm{mL}(P=0.0002)$, although peripheral infection was not significantly associated with plasma IL-6.

Conclusions: These data suggest that plasma cytokine concentrations cannot be used to identify relative changes in the CSF, but that measurement of CSF IL-6 could provide a useful marker of VRI.

Keywords: Cerebrospinal fluid, Cytokines, Infection, Interleukin-6, Markers, Plasma, Subarachnoid haemorrhage, Ventriculostomy

\section{Background}

Experimental and clinical studies of traumatic brain injury and stroke have identified inflammation as an important element of the pathological response [1,2]. In clinical studies, the relationship between clinical status and inflammation has commonly been established by measuring inflammatory markers in plasma or cerebrospinal fluid (CSF) [3-6]. Collection of CSF is rarely indicated clinically in conditions such as ischaemic stroke,

\footnotetext{
*Correspondence: Steve.Hopkins@manchester.ac.uk

${ }^{1}$ The University of Manchester Stroke \& Vascular Centre, Manchester Academic Health Science Centre, Salford Royal Hospitals Foundation Trust, Eccles Old Road, Stott Lane, Salford M6 8HD, UK

Full list of author information is available at the end of the article
}

but is possible in patients with traumatic injury or subarachnoid haemorrhage (SAH), where CSF from the lateral ventricles can be sampled over time in those who, for clinical reasons, have had an external ventricular drain (EVD) inserted. In a number of clinical studies of both stroke and $\mathrm{SAH}$, inflammation within the CNS has been imputed by detection of increased inflammatory markers in plasma. Support for the validity of this derives from clinical studies that have described an association between plasma inflammatory markers and outcome [7-9]. The relationship between inflammatory markers in CSF and plasma is, however, uncertain. Do the increased plasma markers originate primarily in the brain or are they generated peripherally? Could it be that 
plasma markers identify systemic inflammation, or inflammation at the vascular interface, as an important determinant of outcome, relative to inflammation apparent from CSF data? Addressing these questions has important mechanistic implications in terms of understanding the relative importance of central and peripheral inflammation in these conditions.

The primary objective here was therefore to define the relationship between central and peripheral markers of inflammation, following SAH. To achieve this we undertook a prospective study to compare peripheral (plasma) and central (CSF) inflammatory markers in patients with $\mathrm{SAH}$. This was conducted as part of a larger study of delayed cerebral ischaemia in patients with SAH [10] and took advantage of the availability of CSF from patients who had EVDs placed as part of their clinical care. Patients with EVDs are often critically ill and at high risk of infections. Since infection is also a potent inflammatory stimulus, a secondary, retrospective analysis was undertaken to examine the extent to which peripheral infection or ventriculostomy-related infection (VRI) was associated with observed changes in peripheral or central IL-6.

\section{Methods}

\section{Patient inclusion criteria and data collection}

Patients with SAH were eligible for this study if they presented within 7 days of symptom onset to the Greater Manchester Neuroscience Centre at Salford Royal NHS Foundation Trust and required placement of an external ventricular drain (EVD). All but three patients were recruited consecutively from a cohort that is reported in detail elsewhere [10]. After this, three additional patients were recruited consecutively, under the same conditions, since we had a target of at least 20 patients. Four patients only were excluded, on the basis that less than three paired blood and CSF samples were collected. Prior ethical approval was obtained from the Bolton research Ethics Committee, via the National Research Ethics Service, and the University of Manchester Research Ethics Committee. Informed consent was obtained from each patient or their representative.

Adult patients ( $>18$ years) with a confirmed diagnosis of SAH (CT imaging or positive xanthochromia) were identified between January 2004 and June 2006. Eligibility was restricted to those with an underlying aneurysm who underwent formal angiography.

A detailed record of clinical presentation was made. This included presenting features, modified Rankin score, World Federation of Neurosurgeons Scale (WFNS), Fisher grade, Glasgow coma score aneurysm size and location, detailed demographics, infection and medication screen, atherosclerotic risk factors and physiological parameters. Daily clinical assessments included changes in medication,
GCS, temperature, pulse rate, white cell count (WCC) and other evidence of infection.

A $2 \mathrm{~mL}$ CSF sample was collected at time of EVD insertion, where possible, and a concurrent $10 \mathrm{~mL}$ sample of blood was collected into 100 units of pyrogen-free heparin. Samples were cooled to $4^{\circ} \mathrm{C}$ and centrifuged for $15 \mathrm{~min}$ at 2,000 g. Plasma and CSF were decanted and stored at $-70^{\circ} \mathrm{C}$, prior to analysis of inflammatory markers. Clinical assessments were continued on a daily basis and further paired CSF and plasma samples were collected between 07:00 and 11:00, until 7 days after EVD insertion, where possible.

\section{Infection status}

Infection status was determined from retrospective analysis of patient records, including the infection screening and antibiotic records on the study clinical report form. Definition of infection was divided into: (1) Definite: for peripheral infection this required positive culture, plus clinical picture of infection, raised WCC or commencement of antibiotics, while for VRI a positive culture with a clinical picture of infection or commencement of intrathecal antibiotics was necessary; (2) Probable: for peripheral infection this was positive culture in the absence of clinical picture or raised WCC/ CRP, but where antibiotics were not started; or negative culture in the presence of a clinical picture of infection or raised WCC/CRP, where antibiotics were not started. For VRI this was raised CSF WCC: red cell count ratio (>1:700), in the absence of positive culture or starting intrathecal antibiotics, but with a clinical picture of infection; (3) Unlikely: very limited documentation suggesting infection; and (4) No infection: no documentation suggesting infection, or documentation that excluded infection. Only those infections that were present at study entry, diagnosed during collection of samples, or diagnosed up to 2 days post sampling were counted as relevant.

\section{Cytokine assays}

Measurement of interleukin-1ß (IL-1ß), sIL-1R2, TNF- $\alpha$, sTNFR1, sTNFR2 and IL-10 was performed as described previously [9]. IL-1Ra was also measured as described previously [11] The IL-6 assay was undertaken essentially as in Emsley et al. [12], but instead using PeliPair anti-IL-6 antibodies (M9316, Sanquin, Amsterdam, the Netherlands) and development with Zymed steptavidinhorseradish peroxidise conjugate (ZyMax grade, Zymed Laboratories, San Francisco, CA, USA). IL-8 was measured similarly, but using BioSource Cytoset $^{\mathrm{TM}}$ antibodies and streptavidin (Cat. No. CHC1304; BioSource, Nivelles, Belgium). All cytokines were standardised in the laboratory against the latest respective national or international standards (National Institute for Biological Standards and Control, South Mimms, UK). 


\section{Statistical analysis}

Following log transformation, Pearson's correlation was used to calculate the relationship between peripheral and central IL- 6 or IL-8. To identify the relationship between IL-6 concentration and infection, comparison was made between either cases defined as having definite evidence for infection and those with no evidence, or between those with definite or probable evidence for infection and those with no evidence or unlikely infection. Logistic regression was undertaken, both for trend with increasing peak IL-6 values and in respect of a threshold IL-6 concentration, chosen from initial inspection of the data, to determine the odds ratio for association between IL-6 and infection. Since absence of high CSF IL-6 perfectly predicted absence of infection, logistic regression could not be applied as intended and Fisher's 'exact' test was substituted.

\section{Results}

\section{Samples analysed}

Twenty-five patients were recruited to the study. The median age was 53 years (range, 36 to 72 years) and 14 (56\%) were women. The aneurysm was anterior circulation in 20 and posterior circulation in four, $<10 \mathrm{~mm}$ in 20 and, 10 to $25 \mathrm{~mm}$ in four (missing data from one). Sixteen patients underwent coiling, three clipping, one clipping after failed coiling, and four were treated conservatively (missing data from one). The Fisher grade was 3 in 14 and 4 in 11 . The WFNS grade was I in 6, II in 11 , III in 2 , IV in 1 and $\mathrm{V}$ in 5 . The median number of days from ictus to first sample collection was 2 (range, 0 to 10 days). Matched plasma and CSF samples for at least three time points were available from 21 patients.

\section{Cytokines and receptors that were similar, or higher, in} plasma compared to CSF

Concentrations of TNF- $\alpha$ were detectable in $<30 \%$ of CSF samples and $<10 \%$ of plasma samples $(<0.6 \mathrm{pg} / \mathrm{mL}$; not shown). Concentrations of IL- $1 ß$ in plasma were $>2 \mathrm{pg} / \mathrm{mL}$ in only two samples from a single patient. Although 15 CSF samples had concentrations greater than this, and three samples contained between 10 and $40 \mathrm{pg} / \mathrm{mL}$, the mean concentrations were similar to those of plasma (Figure 1). The IL-1 and TNF receptor concentrations, particularly in CSF, were remarkably similar between individuals, as indicated by the relatively small interquartile range. The pattern for IL-1Ra was similar to IL-1ß, although the concentrations were approximately two orders of magnitude higher. A similar pattern was seen with TNFR1 and IL-10, although IL-10 was consistently slightly higher in CSF. For TNFR2 the plasma concentrations were approximately three-fold higher than in CSF and for IL-1R2 the plasma concentrations were about an order of magnitude higher than in CSF.

\section{High CSF IL-6 and IL-8 concentrations compared to plasma}

The IL- 6 and IL- 8 concentrations in CSF were between one and three orders of magnitude higher than in plasma (Figure 2). In most (97\%) paired samples, IL-8

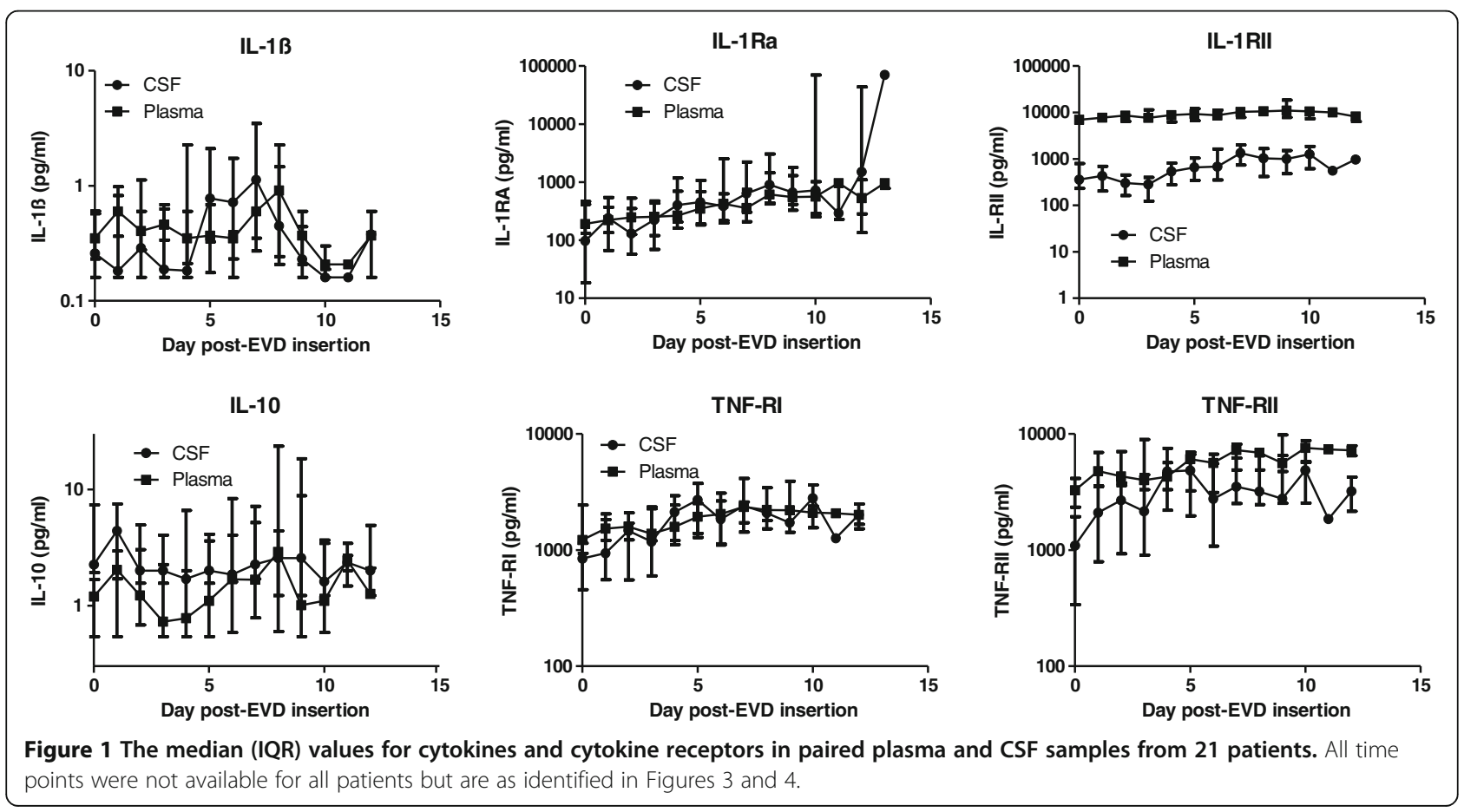


CSF samples remained an order of magnitude greater than plasma (Figure 3). There were very different ratios of plasma and CSF IL-8 between individuals. Correlations for individuals ranged from -0.51 to 0.99 , with a mean of 0.26 .

Although plasma and CSF patterns for IL-6 were remarkably similar to IL-8 in most cases, the order of magnitude difference in concentrations between CSF and plasma was maintained in slightly fewer $(88 \%)$ of the paired samples (Figure 4) and plasma IL-6 concentrations less consistently tracked those in CSF. Correlations for individuals ranged from -0.96 to 0.90 , with a mean of -0.18 .

\section{Association between IL- 6 and infection}

When patients with definite VRI were compared to those with no evidence of VRI (Table 1), the test for increasing odds with increasing IL-6 was incalculable as prediction was perfect. Concentrations of IL- 6 in CSF $>10,000 \mathrm{pg} / \mathrm{mL}$ were significantly associated with VRI (Fisher's: $P<0.001$ ). When cases of probable VRI were combined with definite VRI, and cases of unlikely infection were combined with those having no evidence of VRI, the test for trend was also significant $(P=0.04)$. The association with high IL-6 concentration remained strongly significant (Fisher's: $P<0.001$ ). When peripheral infection (Table 1) was analysed in a similar way, with a cutoff for IL-6 at $30 \mathrm{pg} / \mathrm{mL}$, the odds ratio was not significant for any of the analyses.

\section{Discussion}

Clinical studies of the role of inflammation in brain tissue rely on determinations of biomarkers from clinically accessible fluid compartments. Plasma is clearly the most accessible and can be measured repeatedly to determine changes over time. We have shown that the concentrations of many of the commonly measured markers were similar in plasma and CSF compartments and in some instances plasma markers were higher. Direct comparisons with studies reported previously are problematical, since the assays differ and virtually none make reference to recognised standards. CSF IL- $1 ß$ has been reported as undetectable, or low and stable, in some studies of SAH [3,13], while another study reported it as increased, but in the low picogram range [4], and two others recorded tens to hundreds of picograms per millilitre $[5,6]$. TNF- $\alpha$ has similarly been found to be low or undetectable in some studies $[4,13]$, but increased in others [6,14]. As in our study, IL-1Ra and TNFR1 concentrations have been reported as similar in CSF and plasma or serum, but with an increase after SAH in at least some patients $[14,15]$.

In contrast, CSF concentrations of IL- 6 and IL- 8 were several orders of magnitude greater than those in plasma after SAH. Other studies have consistently found that CSF concentrations of IL-6 and IL- 8 are increased one or two orders of magnitude above those in normal CSF (taken preoperatively for spinal anaesthesia, during elective aneurysm clipping or for diagnostic purposes that subsequently found no inflammatory cause) or plasma values $[3,4,6,13,16,17]$. There was a superficially steady relationship between plasma and CSF concentrations for individual patients, particularly for IL-8. However, the relationship between these compartments in different individuals varies considerably, as indicated by the poor correlation between compartment values for the cohort. This could reflect variability between individuals in the transport of cytokines from CSF to plasma, or simply be due to production occurring discretely in each compartment. The probability of the latter explanation being correct is suggested by the dissociation between plasma and CSF IL-6 following infection.

The absence of a gradient concentration between CSF and plasma for most markers, and the lack of a clear relationship between IL-6 or IL-8 in CSF and plasma, suggest that plasma concentrations of inflammatory markers cannot provide useful information about inflammation in the brain. Several neurosurgical studies have used microdialysis to address this issue, including a study in patients with SAH $[18,19]$. However, apart from
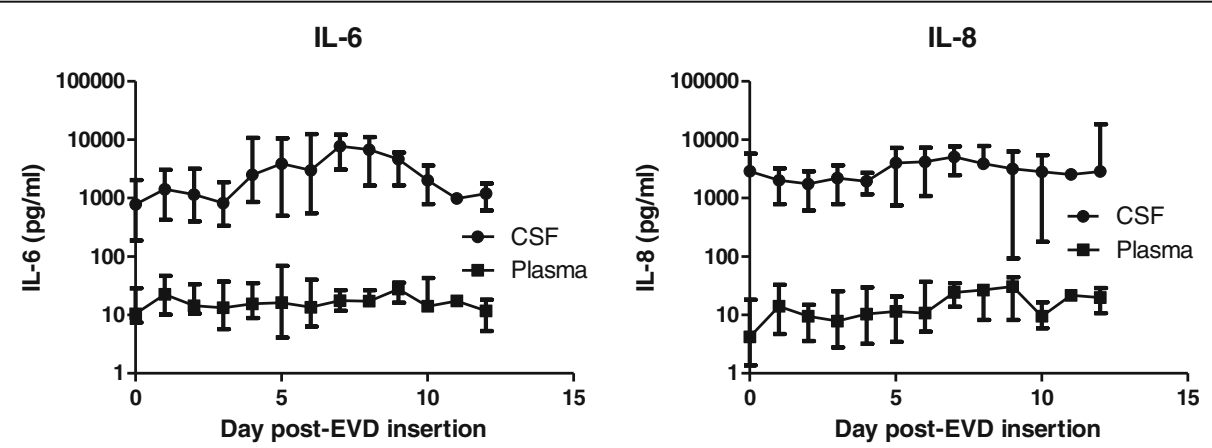

Figure 2 The median (IQR) values for IL-6 and IL-8 in paired plasma and CSF samples from 21 patients. All time points were not available for all patients but are as identified in Figures 3 and 4 . 

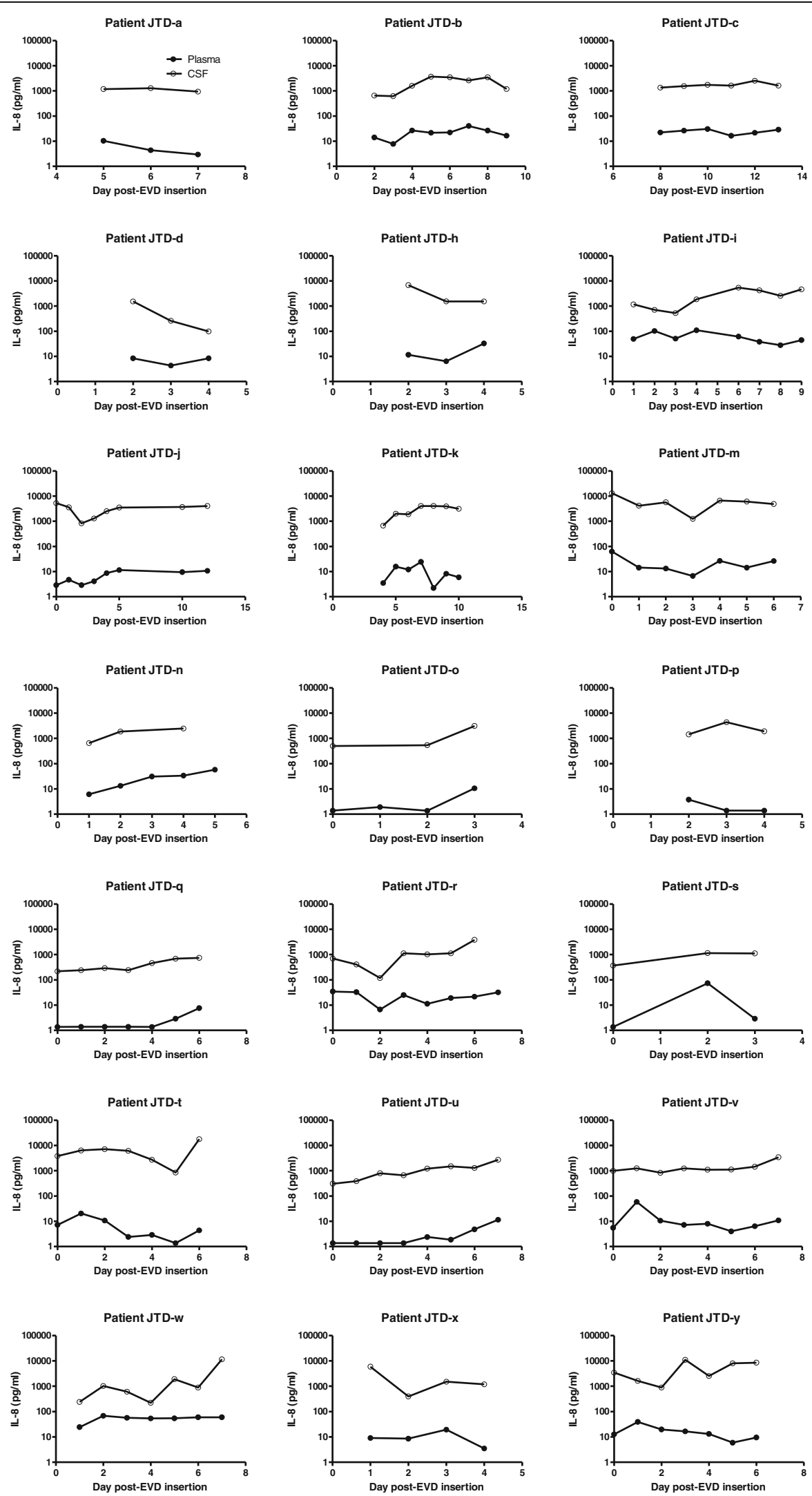

Figure 3 Paired plasma and CSF samples from 21 patients were analysed for IL-8 at the times indicated. 

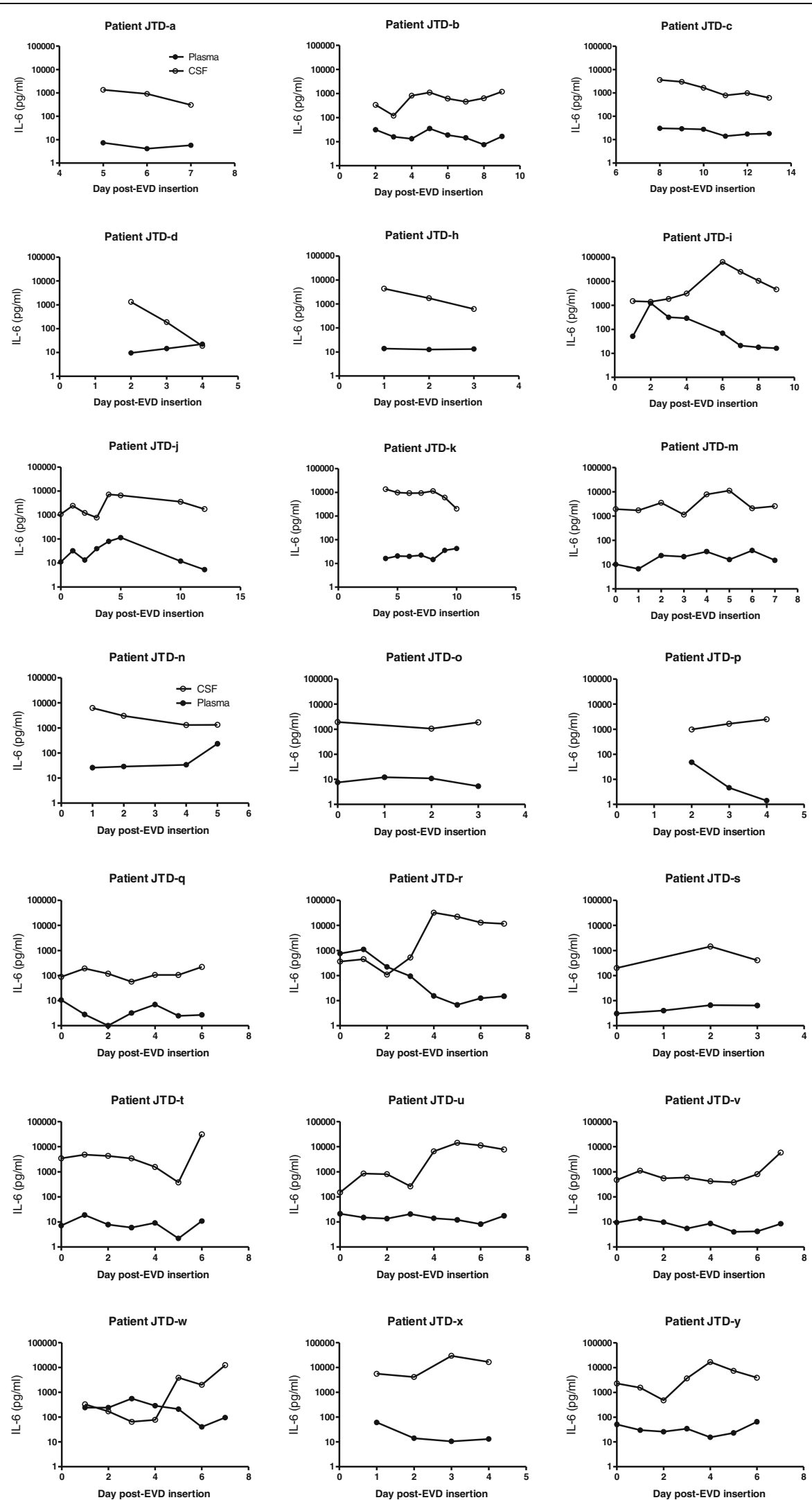

Figure 4 Paired plasma and CSF samples from 21 patients were analysed for IL-6 at the times indicated. 
Table 1 Association between high CSF IL-6 and CSF infection

\begin{tabular}{|c|c|c|}
\hline \multirow[t]{2}{*}{ CNS infection } & \multicolumn{2}{|c|}{ CSF IL-6 } \\
\hline & High $(>10,000 \mathrm{pg} / \mathrm{mL})$ & Low $(<10,000 \mathrm{pg} / \mathrm{mL})$ \\
\hline Definite & $i, k, t, u, x, y$ & \\
\hline Probable & w & \\
\hline No & $m$ & $a, b, c, d, h, j, n, o, p, q, s, v$ \\
\hline Unlikely & $r^{a}$ & \\
\hline
\end{tabular}

the difficulty of placing the dialysis probes such that they provide a representative sample of events taking place in damaged tissue, collected data may reflect tissue damage caused by inserting the probes. It is notable that in a study where a significant change was seen over the collection period (for IL-6, IL-8, macrophage inflammatory protein $1 \beta$ and fibroblast growth factor 2) the highest values were observed in the first $6 \mathrm{~h}$ following probe insertion and these values fell over the next $24 \mathrm{~h}$ [19].

It may be that systemic inflammation and plasma markers are of greater significance than markers detected in the CNS, perhaps because this reflects neurovascular involvement. This is borne out to some extent by the reported associations between systemic inflammation or peripheral inflammatory markers and clinical outcome in situations following brain damage [7,20-23].

Our observations additionally raised questions as to what might be causing some of the changes in cytokine concentration within compartments and altering the relationship between central and peripheral concentrations, particularly for IL- 6 . After reviewing the clinical report forms and patient notes the most consistent other event occurring post-EVD insertion was infection. It is noteworthy that rather little mention is made of infection in study reports or commentaries on the association between cytokines or inflammation following $\mathrm{SAH}$. Infection is a classic activator of inflammation and immunity, and bacterial products are widely used to activate inflammatory responses in experimental settings [24-27]. Ignoring the impact that infection may have on measures of inflammation seems surprising in

Table 2 Association between high plasma IL-6 and peripheral infection

\begin{tabular}{lll}
\hline Peripheral infection & \multicolumn{2}{c}{ Plasma IL-6 } \\
\cline { 2 - 3 } & High $(>\mathbf{3 0} \mathbf{~ p g} / \mathbf{m L})$ & Low $(<\mathbf{3 0} \mathbf{~ g g} / \mathbf{m L})$ \\
\hline Definite & $b, c, i, j, m, n, r, w$ & $s, t, u, v$ \\
\hline Probable & $k$ & \\
\hline No & $p, x$ & $h, q$ \\
\hline Unlikely & $y$ & $a, d, o^{a}$ \\
\hline
\end{tabular}

${ }^{\text {aPatient letters correspond to those in Figures } 3 \text { and } 4 .}$ any study of inflammatory markers, but perhaps particularly in conditions where infection is prevalent. Quite apart from its potential to induce production of cytokines, infection could modify cytokine production occurring as a result of the underlying pathology or might independently have an important influence on clinical outcome. Infection therefore has the potential to obscure relationships between a clinical condition and inflammation, or to suggest associations where both outcome and inflammatory markers are independently influenced by infection.

Depending upon the assessment measures used, infection could potentially alter clinical outcome in several ways. It may influence outcome by directly affecting body systems not threatened by the underlying clinical condition of interest, or modify metabolic functions, including vascular or renal function, such that the primary pathology progresses more rapidly. Alternatively, the infectious stimulus may exacerbate inflammatory responses associated with the condition being investigated. In this respect, several studies have shown that infectious stimuli increase the severity of CNS ischaemic pathology attributable to inflammation $[28,29]$.

Since each of the measured markers has been associated with inflammation it might be expected that most would be similarly affected by infection. Considering the remarkably similar production pattern of IL-6 and IL-8 in these SAH patients, it is perhaps particularly surprising that IL-8 production was quite resistant to modification by infection. Induction of IL-6 seemed particularly sensitive to infection in the patients studied and these data suggest that monitoring IL- 6 might be used as a marker of infection.

The association between IL- 6 and peripheral infection has been well described ([25-27,30,31] and has been indicated as of value in diagnosis [32-34]. It is important to note that ours was a post-hoc analysis, driven by observed data, and therefore likely to overstate the association. Nevertheless, the strength of association is striking and merits further confirmatory studies. Elsewhere, CSF cytokines have been evaluated to diagnose infection in a variety of neurosurgical conditions and IL-1 and TNF were suggested to be of use, whereas IL- 6 and IL- 8 were not [35]. This contrasts with our study, where IL-1 or TNF were barely detectable and only IL-6 production was consistently modified by infection.

At least three other studies have considered the value of monitoring CSF IL- 6 to aid the diagnosis of VRI. Of these, measurement of CSF in four pre-term infants with hydrocephalus suggested that it may be useful [36]. Analysis of IL-6 in 21 adult patients with EVDs concluded that IL-6 was not of value [37]. However, a subsequent study in 75 patients with EVDs concluded that IL-6 had 
significant value for predicting VRI 1 day before other diagnostic markers [38].

The apparent lack of consensus on how VRI is diagnosed highlights the need for an improved method for discrimination. Even positive culture is not definitive and may be identified as due to catheter colonisation or contamination in the majority of cases [39]. Similarly, lack of consensus on use of prophylactic antibiotic cover during use of intracranial monitors and EVDs [40], combined with current concern over inappropriate use of antibiotics, identifies the need for improved, early identification of infection. This study suggests that there would be value in clarifying whether rapid IL-6 measurement may provide a sufficiently sensitive and specific marker to identify VRI early and make a better informed decision on antibiotic use.

\section{Conclusions}

The minor differences between some CSF and plasma cytokines and receptors we identified, combined with the relatively short half-lives of plasma cytokines, do not support the concept that plasma markers are primarily derived from the brain. Further, in the case of cytokines such as IL-6 and IL-8, where there is a significant difference in CSF and plasma concentrations, and these differences are relatively stable within individuals, the variability of the difference between individuals means that it is not possible to deduce differences in CSF concentrations from those observed in plasma. Infection has an important influence on cytokine production, particularly in the case of IL- 6 . The data suggest that account should be taken of infection in patients where inflammatory markers are being evaluated and that IL-6 may be a valuable early indicator of CSF infection in patients with EVDs.

\section{Abbreviations}

CSF: Cerebrospinal fluid; EVD: External ventricular drain; IL: Interleukin; Ra: Receptor antagonist; SAH: Subarachnoid haemorrhage; TNF: Tumour necrosis factor; VRI: Ventriculostomy-related infection.

\section{Competing interests}

The authors declare that they have no competing interests.

\section{Authors' contributions \\ SJH, AV, NJR and PT conceived the study and designed the protocols with CJM and ATK. Patients samples and details were collected by CJM, JG and $\mathrm{SH}$. Samples were analysed by $\mathrm{MH}$ and SS, and data was analysed and interpreted by SJH, CJM, NS, MH, SS, HP, AV,SH and ATK. The manuscript was drafted by SJH, with input from all other authors, who also approved the final manuscript.}

\section{Acknowledgements}

This work was supported by The Sir Jules Thorn Charitable Trust (Ref: 02/JTA).

\section{Author details}

${ }^{1}$ The University of Manchester Stroke \& Vascular Centre, Manchester Academic Health Science Centre, Salford Royal Hospitals Foundation Trust, Eccles Old Road, Stott Lane, Salford M6 8HD, UK. ${ }^{2}$ Faculty of Life Sciences,
University of Manchester, AV Hill Building, University of Manchester, Oxford Road, Manchester M13 9PT, UK. 'epartment of Neurosurgery, Leeds General Infirmary, Great George Street, Leeds LS1 3EX, UK.

Received: 1 October 2012 Accepted: 13 November 2012

Published: 23 November 2012

\section{References}

1. Denes A, Thornton P, Rothwell NJ, Allan SM: Inflammation and brain injury: acute cerebral ischaemia, peripheral and central inflammation. Brain Behav Immun 2010, 24:708-723.

2. Lakhan S, Kirchgessner A, Hofer M: Inflammatory mechanisms in ischemic stroke: therapeutic approaches. J Transl Med 2009, 7:97.

3. Osuka K, Suzuki Y, Tanazawa T, Hattori K, Yamamoto N, Takayasu M, Shibuya M, Yoshida J: Interleukin- 6 and development of vasospasm after subarachnoid haemorrhage. Acta Neurochir 1998, 140:943-951.

4. Fassbender K, Hodapp B, Rossol S, Bertsch T, Schmeck J, Schutt S, Fritzinger M, Horn P, Vajkoczy P, Kreisel S, Brunner J, Schmiedek P, Hennerici M: Inflammatory cytokines in subarachnoid haemorrhage: association with abnormal blood flow velocities in basal cerebral arteries. J Neurol Neurosurg Psychiatry 2001, 70:534-537.

5. Hendryk S, Jarzab B, Josko J: Increase of the IL-1 beta and IL-6 levels in CSF in patients with vasospasm following aneurysmal SAH. Neuroendocrinology Letters 2004, 25:141-147.

6. Kwon KY, Jeon BC: Cytokine levels in cerebrospinal fluid and delayed ischemic deficits in patients with aneurysmal subarachnoid hemorrhage. J Korean Med Sci 2001, 16:774-780.

7. Smith CJ, Emsley CA, Gavin CM, Georgiou RF, Vail A, Barberan EM, del Zoppo GJ, Hallenbeck JM, Rothwell NJ, Hopkins SJ, Tyrrell PJ: Peak plasma interleukin- 6 and other peripheral markers of inflammation in the first week of ischaemic stroke correlate with brain infarct volume, stroke severity and long-term outcome. BMC Neurol 2004, 4:2.

8. Fassbender K, Rossol S, Kammer T, Daffertshofer M, Wirth S, Dollman M, Hennerici M: Proinflammatory cytokines in serum of patients with acute cerebral-ischemia - kinetics of secretion and relation to the extent of brain-damage and outcome of disease. J Neurol Sci 1994, 122:135-139.

9. Emsley H, Smith C, Gavin C, Georgiou R, Vail A, Barberan E, Illingworth K, Scarth S, Wickramasinghe V, Hoadley M, Rothwell NJ, Tyrrell PJ, Hopkins SJ: Clinical outcome following acute ischaemic stroke relates to both activation and autoregulatory inhibition of cytokine production. BMC Neurol 2007, 7:5.

10. McMahon CJ, Hopkins S, Vail A, King AT, Smith D, Illingworth KJ, Clark S: Rothwell NJ. Tyrrell PJ: Inflammation as a predictor for delayed cerebral ischaemia after aneurysmal subarachnoid haemorrhage. J Neurointerv Surg; 2012 Oct 11 [Epub ahead of print].

11. Emsley HCA, Smith CJ, Georgiou RF, Vail A, Hopkins SJ, Rothwell NJ, Tyrrell PJ, Investigators ftI-riAS: A randomised phase II study of interleukin-1 receptor antagonist in acute stroke patients. I Neurol Neurosurg Psychiatry 2005, 76:1366-1372.

12. Emsley HCA, Smith CJ, Gavin CM, Georgiou RF, Vail A, Barberan EM, Hallenbeck JM, del Zoppo GJ, Rothwell NJ, Tyrrell PJ, Hopkins SJ: An early and sustained peripheral inflammatory response in acute ischaemic stroke: relationships with infection and atherosclerosis. J Neuroimmunol 2003, 139:93-101.

13. Kikuchi T, Okuda Y, Kaito N, Abe T: Cytokine production in cerebrospinal fluid after subarachnoid hemorrhage. Neurol Res 1995, 17:106-108.

14. Mathiesen T, Edner G, Ulfarsson E, Andersson B: Cerebrospinal fluid interleukin-1 receptor antagonist and tumor necrosis factor-alpha following subarachnoid hemorrhage. J Neurosurg 1997, 87:215-220

15. Gruber A, Rossler K, Graninger W, Donner A, Illievich MU, Czech T: Ventricular cerebrospinal fluid and serum concentrations of sTNFR-I, IL-1 ra, and IL-6 after aneurysmal subarachnoid hemorrhage. J Neurosurg Anesthesiol 2000, 12:297-306.

16. Gaetani P, Tartara F, Pignatti P, Tancioni F, Baena RRY, De Benedetti F: Cisternal CSF levels of cytokines after subarachnoid hemorrhage. Neurol Res 1998, 20:337-342.

17. Mathiesen T, Andersson B, Loftenius A, von Holst H: Increased interleukin-6 levels in cerebrospinal fluid following subarachnoid hemorrhage. J Neurosurg 1993, 78:562-567. 
18. Graetz D, Nagel A, Schlenk F, Sakowitz O, Vajkoczy P, Sarrafzadeh A: High ICP as trigger of proinflammatory IL-6 cytokine activation in aneurysmal subarachnoid hemorrhage. Neurol Res 2010, 32:728-735

19. Mellergard P, Aneman O, Sjogren F, Pettersson P, Hillman J: Changes in extracellular concentrations of some cytokines, chemokines, and neurotrophic factors after insertion of intracerebral microdialysis catheters in neurosurgical patients. Neurosurgery 2008, 62:151-157.

20. Dhar $\mathrm{R}$, Diringer $\mathrm{M}$ : The burden of the systemic inflammatory response predicts vasospasm and outcome after subarachnoid hemorrhage. Neurocrit Care 2008, 8:404-412.

21. Shenhar-Tsarfaty S, Assayag EB, Bova I, Shopin L, Fried M, Berliner S, Shapira I, Bornstein NM: Interleukin- 6 as an early predictor for one-year survival following an ischaemic stroke/transient ischaemic attack. Int J Stroke 2010, 5:16-20

22. Whiteley W, Jackson C, Lewis S, Lowe G, Rumley A, Sandercock P, Wardlaw J, Dennis M, Sudlow C: Inflammatory markers and poor outcome after stroke: a prospective cohort study and systematic review of interleukin-6. PLOS Med 2009, 6:e1000145.

23. Woiciechowsky C, Schöning B, Cobanov J, Lanksch WR, Volk H-D, Döcke W-D: Early IL-6 plasma concentrations correlate with severity of brain injury and pneumonia in brain-injured patients. J Trauma 2002, 52:339-345.

24. Arend WP, Maseoni RJ: Characteristics of bacterial lipopolysaccharide induction of interleukin 1 synthesis and secretion by human monocytes. Clin Exp Immunol 1986, 64:656-664.

25. Calandra T, Gerain J, Heumann D, Baumgartner JD, Glauser MP: High circulating levels of interleukin- 6 in patients with septic shock Evolution during sepsis, prognostic value, and interplay with other cytokines. Am J Med 1991, 91:23-29.

26. Helfgott DC, Tatter SB, Santhanam U, Clarick RH, Bhardwaj N, May LT, Sehgal B: Multiple forms of IFN-á2 / IL-6 in plasma and body fluids during acute bacterial infection. J Immunol 1989, 142:948-953.

27. Jirik FR, Poder TJ, Hirano T, Kishimoto T, Loskutoff DJ, Carson DA, Lotz DA: Bacterial lipopolysaccharide and inflammatory mediators augment IL-6 secretion by human endothelial cells. J Immunol 1989, 142:144-147.

28. Emsley HCA, Hopkins SJ: Acute ischaemic stroke and infection: recent and emerging concepts. Lancet Neurol 2008, 7:341-353.

29. McColl BW, Rothwell NJ, Allan SM: Systemic inflammatory stimulus potentiates the acute phase and CXC chemokine responses to experimental stroke and exacerbates brain damage via interleukin-1and neutrophil-dependent mechanisms. J Neurosci 2007, 27:4403-4412.

30. Fong Y, Moldawer LL, Marano MA, Wei H, Tatter SB, Clarick RH, Santhanam U, Sherris D, May LT, Sehgal PB, Lowry SF: Endotoxemia elicits increasted circulating 2-IFN / IL-6 in man. J Immunol 1989, 142:2321-2324.

31. Hack CE, Degroot ER, Feltbersma RJF, Niujens JH, Vanschijndel RJMS, Eerenbergbelmer AJM, Thijs LG, Aarden LA: Increased plasma levels of interleukin-6 in sepsis. Blood 1989, 74:1704-1710.

32. Foulon W, Van Liedekerke D, Demanet C, Decatte L, Dewaele M, Naessens $A$ : Markers of infection and their relationship to preterm delivery. Am J Perinatol 1995, 12:208-211.

33. Moscovitz H, Shofer F, Mignott H, Behrman A, Kilpatrick L: Plasma cytokine determinations in emergency department patients as a predictor of bacteremia and infectious disease severity. Crit Care Med 1994, 22:1102-1107.

34. Rego MAC, Martinez FE, Elias J, Mussi-Pinhata MM: Diagnostic value of interleukin-6 and C-reactive protein on early onset bacterial infection in preterm neonates with respiratory distress. J Perinat Med 2010, 38:527-533.

35. López-Cortés LF, Marquez-Arbizu R, Jimenez-Jimenez LM, Jimenez-Mejías E, Caballero-Granado FJ, Rey-Romero C, Polaina M, Pachón J: Cerebrospinal fluid tumor necrosis factor-[alpha], interleukin-1[beta], interleukin-6, and interleukin-8 as diagnostic markers of cerebrospinal fluid infection in neurosurgical patients. Crit Care Med 2000, 28:215-219.

36. Baumeister FAM, Pohl-Koppe A, Hofer M, Kim JO, Weiss M: IL-6 in CSF during ventriculitis in preterm infants with posthemorrhagic hydrocephalus. Infection 2000, 28:234-236.

37. Schade RP, Schinkel J, Roelandse FWC, Geskus RB, Visser LG, van Dijk MC Voormolen JHC, van Pelt H, Kuijper EJ: Lack of value of routine analysis of cerebrospinal fluid for prediction and diagnosis of external drainage-related bacterial meningitis. J Neurosurg 2006, 104:101-108.
38. Schoch B, Regel JP, Nierhaus A, Wichert M, Mueller OM, Sandalcioglu IE, Mann K, Stolke D: Predictive value of intrathecal interleukin- 6 for ventriculostomy-related infection. Cen Eur Neurosurg 2008, 69:80-86.

39. Bota DP, Lefranc F, Vilallobos HR, Brimioulle S, Vincent J-L: Ventriculostomy-related infections in critically ill patients: a 6-year experience. J Neurosurg 2005, 103:468-472.

40. Prabhu VC, Kaufman HH, Voelker JL, Aronoff SC, Niewiadomska-Bugaj M, Mascaro S, Hobbs GR: Prophylactic antibiotics with intracranial pressure monitors and external ventricular drains: a review of the evidence. Surg Neurol 1999, 52:226-237.

doi:10.1186/1742-2094-9-255

Cite this article as: Hopkins et al:: Cerebrospinal fluid and plasma cytokines after subarachnoid haemorrhage: CSF interleukin- 6 may be an early marker of infection. Journal of Neuroinflammation 2012 9:255.

\section{Submit your next manuscript to BioMed Central and take full advantage of:}

- Convenient online submission

- Thorough peer review

- No space constraints or color figure charges

- Immediate publication on acceptance

- Inclusion in PubMed, CAS, Scopus and Google Scholar

- Research which is freely available for redistribution 